\title{
Estimation of Thermodynamic and Interfacial Parameters of Metallic Materials by Molecular Dynamics Simulations
}

\author{
Yasushi Shibuta* \\ Department of Materials Engineering, The University of Tokyo, Tokyo 113-8656, Japan
}

The range of application of molecular dynamics (MD) simulations is rapidly expanding owing to the recent advance in high-performance computing. Since only the coordinate and velocity of atoms in the system are directly obtained from MD simulations, it is important to correctly understand how the coordinate and velocity of atoms are converted into thermodynamic and interfacial properties. Here, MD-based techniques for estimating the thermodynamic and interfacial properties of metallic materials are assessed by considering practical examples of the melting point of a pure metal, the solidus and liquidus compositions of a binary alloy, the grain boundary energy, the solid-liquid energy, and the kinetic coefficient. [doi:10.2320/matertrans.ME201712]

(Received May 28, 2018; Accepted December 6, 2018; Published January 11, 2019)

Keywords: molecular dynamics simulation, melting, solidification, microstructure, solid-liquid interface, grain boundary, kinetic coefficient

\section{Introduction}

Owing to the recent advance in high-performance computing, the range of application of atomistic simulations is rapidly expanding. ${ }^{1,2)}$ The motion of all atoms in a submicrometer-scale cube can now be directly handled in molecular dynamics (MD) simulations ${ }^{3)}$ and large-scale MD simulations with up to a billion atoms have been applied in the manufacturing process of structural materials. ${ }^{3-6)}$ On the other hand, another critical problem has emerged as the number of atoms in MD simulations has increased. That is, a high computational cost is required not only for the main MD simulation but also for the postanalysis processing of the simulation data. ${ }^{3)}$ In principle, only the coordinate and velocity of atoms are directly obtained from MD simulations, which are translated into macroscopic physical quantities. Some basic intensive variables such as temperature and pressure can be derived exactly from the coordinate and velocity of atoms since their relationships are clearly defined in thermodynamics in conjunction with statistical mechanics. Moreover, some mechanical properties of perfect crystalline materials are clearly defined and reproduced well in MD simulations since these properties strongly correlate with the shape and depth of the well of interatomic potential. Therefore, MD simulations have been widely employed to examine mechanical properties ${ }^{7)}$ such as dislocation, ${ }^{8)}$ fracture, ${ }^{9)}$ plastic deformation, ${ }^{10,11)}$ and stacking fault tetrahedra. $^{12)}$

On the other hand, it is not straightforward to deterministically define the entropy of a system from the coordinate and velocity of atoms since the number of states cannot be defined in principle for a system governed by the classical equation of motion. ${ }^{13)}$ Therefore, it is essential to employ an alternative technique that reproduces the desired ensemble. ${ }^{13)}$ For example, a Monte Carlo (MC) simulation, in which the chemical potential is specified, is often used to reproduce the desired ensemble. Thermodynamic integration is also useful, in which the free energy is converted from quantities correlated with the partition function. However, many

*Corresponding author, E-mail: shibuta@material.t.u-tokyo.ac.jp simulations must be performed to obtain the statistical average.

In addition to conventional $\mathrm{MD}$ and $\mathrm{MC}$ simulations, recent progress in collaboration with techniques from data science including computational screening, ${ }^{14,15)}$ neural network computation, ${ }^{16}$ machine learning ${ }^{17}$ and data assimilation $^{18,19)}$ is opening a new window of atomistic simulations. As techniques based on the data science permeate computational metallurgy, it becomes increasingly important to ensure the feasibility of simulation results from a metallurgical viewpoint. To this end, techniques for parameter estimation from MD simulations are comprehensively assessed in this paper. In particular, the thermodynamic and interfacial properties of metallic materials are focused on since these properties directly affect the manufacturing process of structural materials.

\section{Molecular Dynamics Simulation}

\subsection{Brief summary of simulation methodology}

An MD simulation is a deterministic method of atomistic simulation, in which a full set of the coordinate and velocity of all atoms in a target material is determined by numerically solving the Newton's equation of motion. Once the initial configuration and the definition of the interatomic force are given, the coordinate and velocity of all atoms at every time step are determined explicitly, from which macroscopic physical properties are derived. Since a numerical technique for the integration of Newton's equation of motion has already been established, ${ }^{20)}$ the following two points are key issues: (i) how to define the interatomic force and (ii) how to estimate physical properties only from the coordinate and velocity of atoms.

In principle, the interatomic force is defined from the electronic states of the atomistic configuration. An MD simulation using the interatomic force derived directly from a quantum-based calculation is called an ab initio MD simulation or a first-principles MD simulation. ${ }^{21)}$ Although an ab initio MD simulation can treat the interatomic force precisely without any empirical parameters, it requires a high computational cost. Therefore, the interatomic force is often 
derived from an empirical function called the interatomic potential (or the force field). An MD simulation using the interatomic potential is called a classical MD simulation. Note that "MD simulation" without the therm ab initio or first-principles conventionally refers to a classical MD simulation. It is essential to employ an ab initio MD simulation for quantitative discussion of the elementary steps of chemical reactions including the charge transfer. ${ }^{22-27)}$ On the other hand, it is at present reasonable to employ a classical MD simulation for most metallurgical processes in which the cooperative motion of many atoms is dominant, such as phase transformation, nucleation, and grain growth. In summary, it is important to employ an appropriate methodology depending on the purpose of the study.

\subsection{Interatomic potential and force}

In general, interatomic potentials are classified into two types: the pairwise potential and the many-body potential. In the pairwise potential, the total potential energy of a system, $U$, is described by the interatomic distance $r_{i j}$ between atoms $i$ and $j$ as

$$
U=\frac{1}{2} \sum_{i} \sum_{j} U_{i j}\left(r_{i j}\right)
$$

Representative pairwise potentials are the Lennard-Jones potential, ${ }^{28)}$ which is widely used in simplified models, and the Morse potential ${ }^{29}$ ) for covalent and metal bonds. For example, parameter sets of the Morse potential have been proposed for many metallic bonds. ${ }^{30)}$ However, the pairwise potential cannot correctly reproduce the anisotropy in mechanical properties. ${ }^{31)}$

Therefore, interatomic potentials including many-body effects have been widely proposed. The most popular one for metals and alloys is the embedded atom method (EAM) potential. ${ }^{32)}$ The EAM potential is generally described by a combination of the pair term $V_{i j}$ between atoms $i$ and $j$ and the embedded function $F$ as

$$
\begin{gathered}
U=\frac{1}{2} \sum_{i} \sum_{j} V_{i j}\left(r_{i j}\right)+\sum_{i} F\left(\rho_{i}\right), \\
\rho_{i}=\sum_{j \neq i} \varphi_{i j}\left(r_{i j}\right),
\end{gathered}
$$

where $\rho_{i}$ is the electron density around atom $i$, which is the sum of the electron densities from the neighboring atoms $j$ of atom $i, \varphi\left(r_{i j}\right)$. Since Daw and Baskes ${ }^{32)}$ proposed the original form of the EAM functions, many modified versions have been proposed such as the generalized EAM (GEAM) ${ }^{33)}$ for multicomponent alloys and the modified EAM (MEAM) ${ }^{34)}$ including angular terms. On the other hand, the StillingerWeber potential ${ }^{35)}$ has often been used for materials with the diamond structure such as silicon and germanium. This potential consists of a combination of pairwise and angular terms (three-body term) as

$$
U=\frac{1}{2} \sum_{i} \sum_{j} V_{i j}\left(r_{i j}\right)+\sum_{i<j<k} \phi_{3}\left(\mathbf{r}_{i}, \mathbf{r}_{j}, \mathbf{r}_{k}\right),
$$

where $\mathbf{r}_{i}, \mathbf{r}_{j}$, and $\mathbf{r}_{k}$ are the positions of atoms $i, j$, and $k$, respectively. Bond-order type potentials ${ }^{36,37)}$ are also commonly used for covalent systems including silicon and carbon. Moreover, interatomic potentials including those for the chemical reactions and charge transfer, as typified by the ReaxFF ${ }^{38)}$ and $\mathrm{COMB}^{39)}$ potentials, have recently been developed.

The force acting on atom $i, \mathbf{F}_{i}$, is defined as the gradient of the interatomic potential,

$$
\mathbf{F}_{i}=-\frac{\partial U\left(\mathbf{r}_{i}\right)}{\partial \mathbf{r}_{i}} .
$$

The analytical solution of the interatomic force is easily derived for the pairwise potential, whereas it is not straightforward to derive it from a complicated many-body potential. Recently, a large amount of numerical data on interatomic potentials has been made available in databases (for example, the Interatomic Potentials Repository Project ${ }^{40)}$ ). The utilization of these databases in combination with open-source software such as the LAMMPS molecular dynamics simulator ${ }^{41)}$ can greatly reduce the time and cost of MD simulations. In this study, LAMMPS ${ }^{41)}$ and the database from Interatomic Potential Repository Project ${ }^{40}$ are utilized for the simulations in Sections 3.1, 3.2, and 3.3, whereas our own code is employed for the simulations in Sections 3.4, 3.5, and 3.6.

\subsection{Temperature and pressure in atomistic system}

In an MD simulation, the law of equipartition of energy holds for the motion of atoms since the dynamics of atoms is governed by Newton's law of motion. According to the law of equipartition of energy, the average kinetic energy is $k_{B} T / 2$ ( $k_{B}$, Boltzmann constant; $T$, temperature) per degree of freedom in thermal equilibrium. This gives the following relation:

$$
\left\langle\sum_{i=1}^{n} \frac{1}{2} m_{i} \mathbf{v}_{i}^{2}\right\rangle=\frac{3}{2} n k_{B}\langle T\rangle,
$$

where $m_{i}$ and $\mathbf{v} i$ are the mass and velocity of atom $i$, respectively, and $n$ is the total number of atoms in the system. The brackets \langle\rangle represent the ensemble average. Therefore, the temperature of the system is defined using the velocity of atoms as

$$
\langle T\rangle=\frac{1}{3 n k_{B}}\left\langle\sum_{i=1}^{n} m_{i} \mathbf{v}_{i}^{2}\right\rangle .
$$

In the same manner, the instantaneous temperature of atom $i$ can be defined as

$$
T_{i}=\frac{m_{i} \mathbf{v}_{i}^{2}}{3 k_{B}} .
$$

In reality, the instantaneous temperature has a distribution even when the average temperature in the system is $\langle T\rangle$. For example, the Maxwell-Boltzmann distribution holds for the distribution of the instantaneous temperature of atoms in the equilibrium gas state. Moreover, the pressure in the system is defined from the virial theorem as

$$
\langle P\rangle=\frac{n k_{B}\langle T\rangle}{V}+\frac{1}{3 V}\left\langle\sum_{i=1}^{n} \mathbf{r}_{i} \cdot \mathbf{F}_{i}\right\rangle,
$$

where $V$ is the volume of the system. By substituting eqs. (5) and (7) into eq. (9), the pressure of the system can be defined using the coordinate and velocity of the atoms in the system as 


$$
\langle P\rangle=\frac{1}{3 V}\left(\left\langle\sum_{i=1}^{n} m_{i} \mathbf{v}_{i}\right\rangle-\left\langle\sum_{i=1}^{n} \mathbf{r}_{i} \cdot \frac{\partial U\left(\mathbf{r}_{i}\right)}{\partial \mathbf{r}_{i}}\right\rangle\right) .
$$

In general, the fluctuation in pressure is larger than that in temperature in practical simulations.

\section{Estimation of Thermodynamic and Interfacial Pa- rameters}

\subsection{Melting point of pure metal}

In this section, practical examples of the estimation of some thermodynamic and interfacial properties related to metallurgy are introduced. The melting point of pure metal is usually estimated from the equilibrium temperature, at which the planar solid-liquid interface does not move in a solid-liquid biphasic system. ${ }^{42-44)}$ Figure 1(a) shows the solid-liquid biphasic Ni system $(96,000$ atoms in a cell of $70.4 \times 70.4 \times 311.2 \AA$ ) expressed by the EAM potential of Purja Pun and Mishin. ${ }^{45)}$ The prepared system is first relaxed with the isobaric-isothermal ensemble (called the number, pressure and temperature (NPT)-constant ensemble) for $50 \mathrm{ps}$ at 1650,1675 , and $1700 \mathrm{~K}$. Then, the system is relaxed with the isenthalpic-isobaric ensemble (called the number, pressure and enthalpy (NPH)-constant ensemble) for $450 \mathrm{ps}$. The pressure is maintained at $0 \mathrm{~Pa}$ in all the processes. The time step for the integration of Newton's equation of motion is set to $1 \mathrm{fs}$ throughout this paper. Figure 1(b) shows the time dependence of the temperature of the system. When the initial temperature is lower than the melting point, solidification occurs at the solid-liquid interface. Since the solidification releases latent heat, the temperature of the system increases until it reaches the melting point. On the other hand, melting occurs when the initial temperature is higher than the melting point. The temperature of the system
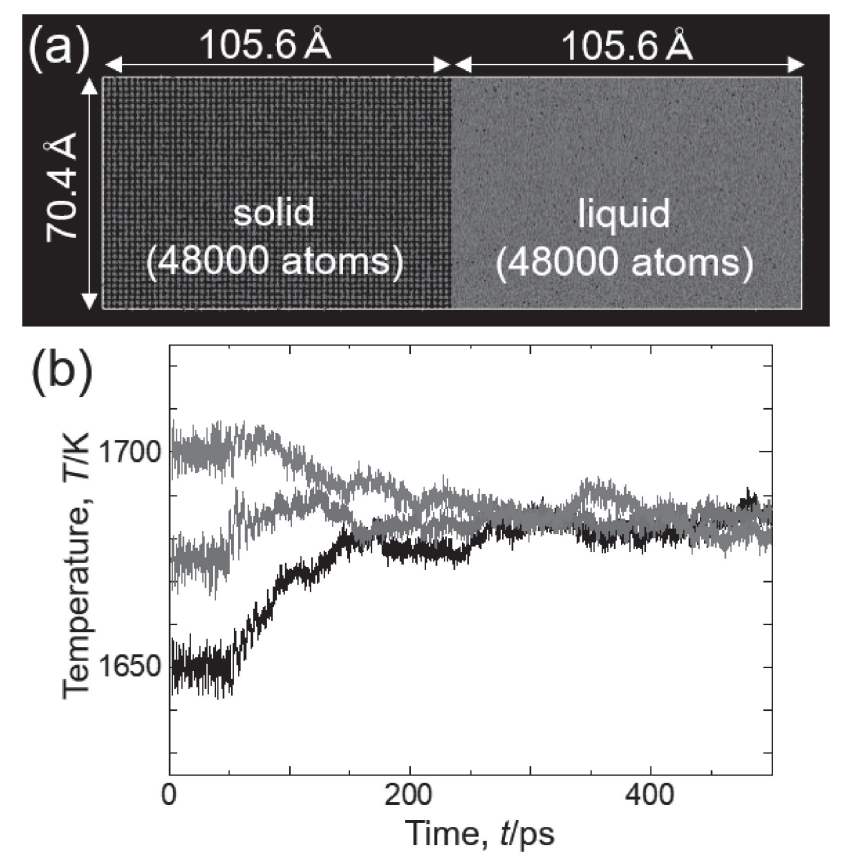

Fig. 1 (a) Snapshot of the solid-liquid biphasic Ni system for estimating the melting point for the interatomic potential employed in the MD simulation. The periodic boundary condition is employed in all directions. (b) Time dependence of the temperature of the system. decreases during the melting owing to the absorption of latent heat. Therefore, the temperature to which the system converges is regarded as its melting point. As shown in Fig. 1(b), the system temperatures converge to approximately $1685 \pm 10 \mathrm{~K}$, which is regarded as the melting point of $\mathrm{Ni}$ expressed by this EAM potential. Note that the melting point in an MD simulation tends to deviate from the experimental value to. This is mainly due to the difficulty of directly reading the melting point from the interatomic potential curve itself. Therefore, the temperature normalized by the melting point of the interatomic potential is often used for discussion of the temperature effect.

\subsection{Thermodynamic melting and mechanical melting}

Usually, the melting starts from an inhomogeneous region in the crystal such as a surface or a grain boundary. The melting induced by an inhomogeneous region is called thermodynamic melting, which occurs at the melting point. On the other hand, another type of melting is sometimes observed in MD simulations. That is, an infinite perfect crystal in a supercell retains its structure even at the melting point and collapses spontaneously at a temperature above the melting point. This type of melting is called mechanical melting. ${ }^{46}$ Figure 2 shows snapshots of thermodynamic melting and mechanical melting. Perfect Ni crystals $(187,500$ atoms, $88.0 \times 88.0 \times 264.0 \AA)$ are prepared under two periodic boundary conditions: (i) in all directions (i.e., an infinite perfect crystal) and (ii) in only the $y$ and $z$ directions (i.e., a crystal with surfaces in the $x$ direction). Then, these crystals are relaxed for $50 \mathrm{ps}$ at high temperatures near the melting point defined in Fig. 1(b). Figure 2 shows these crystals after the relaxation at various temperatures. When the crystal with surfaces is relaxed at a higher temperature than the melting point $(1685 \mathrm{~K})$, the crystal starts melting at the surface and the solid-liquid interface propagates into the crystal. The solid-liquid interface propagates more rapidly as the temperature increases. This type of melting is thermodynamic melting.

However, the infinite perfect crystal does not melt even at higher temperatures (1800 and $1900 \mathrm{~K}$ ) than the melting point $(1685 \mathrm{~K})$. It is considered that superheating occurs in the infinite perfect crystal owing to the lack of onset of melting. The infinite perfect crystal suddenly homogeneously melts at $2100 \mathrm{~K}$ and mechanically breaks owing to the instability limit of the interatomic bonds. This is regarded as mechanical melting. Since inhomogeneous regions such as the surface and grain boundaries usually exist in practical crystals, thermodynamic melting should occur in reality. Note that mechanical melting occurs virtually in computer simulations when an infinite perfect crystal is employed in a small supercell. On the other hand, solidification usually occurs in an undercooled liquid via nucleation since there is an energy barrier for nucleation due to the effect of solidliquid interfacial energy. Therefore, nucleation is regarded as a thermally activated (i.e., kinetic) process. Thermally activated nucleation from an undercooled melt has been confirmed in an MD simulation, ${ }^{4)}$ in which the graph of the nucleation rate with respect to temperature has a nose shape due to the two competing temperature effects. This is reasonable from both thermodynamic and kinetic viewpoints. 


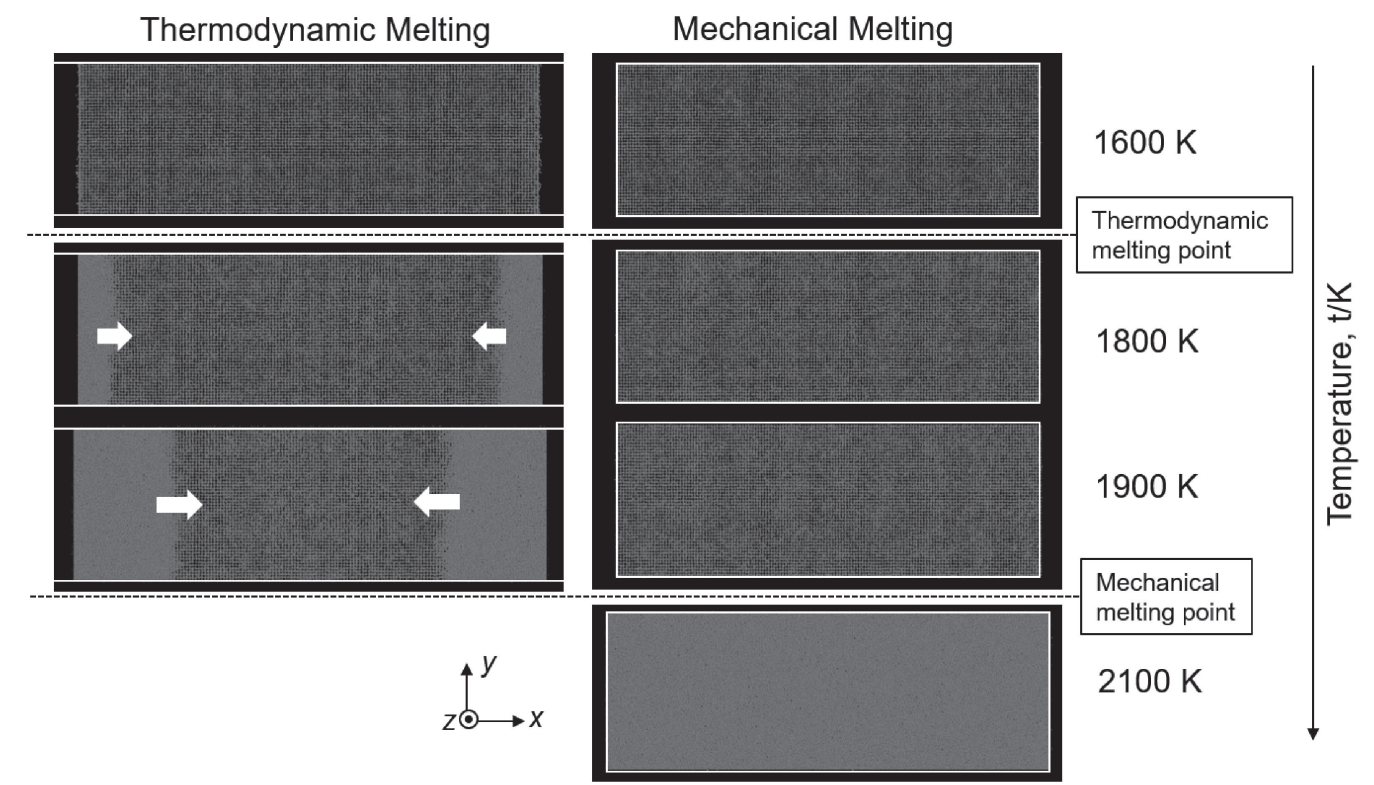

Fig. 2 Snapshots of thermodynamic melting and mechanical melting for Ni crystals after relaxation for $50 \mathrm{ps}$ at high temperatures.

\subsection{Solidus and liquidus compositions of binary alloys}

For alloy systems, solidus and liquidus temperatures are defined instead of the melting point. In accordance with the thermodynamics of materials, ${ }^{47}$ the solidus and liquidus compositions with respect to temperature are defined from the contact points of the common tangent line with the Gibbs free energies of mixing for the solid and liquid phases. Since it is not straightforward to directly estimate the Gibbs free energy of mixing from an MD simulation, the semigrand canonical Monte Carlo (SGCMC) method ${ }^{48-51)}$ is often employed, in which the equilibrium concentration is obtained for the given chemical potential difference $\Delta \mu$. Here, the practical procedure is explained by considering the example of an $\mathrm{Fe}-\mathrm{Cr}$ alloy using the EAM potential defined by Bonny et al. $^{52)}$ SGCMC simulations with various values of $\Delta \mu$ are performed for both solid and liquid systems at $2500 \mathrm{~K}$. The corresponding equilibrium concentrations are obtained from the calculations. Figure 3(a) shows the relationship between $\Delta \mu$ and the equilibrium concentration $X_{\mathrm{Fe}}$ at $2500 \mathrm{~K}$ for both solid and liquid alloys obtained by the SGCMC simulations. Note that the melting points of pure $\mathrm{Fe}$ and $\mathrm{Cr}$ for this potential are $1770 \pm 20$ and $2670 \pm 20 \mathrm{~K}$, respectively. That is, the set temperature, $2500 \mathrm{~K}$, lies between the melting points of $\mathrm{Fe}$ and $\mathrm{Cr}$. Then, the plots obtained from the simulation are fitted to the following equation ${ }^{49)}$ to obtain the fitting parameters $A_{i}(i=0,1, \ldots, n)$.

$$
\Delta \mu\left(X_{\mathrm{Fe}}\right)=k_{B} T \ln \left(\frac{X_{\mathrm{Fe}}}{1-X_{\mathrm{Fe}}}\right)+\sum_{i=0}^{n} A_{i} X_{\mathrm{Fe}}^{i}
$$

A quintic polynomial is employed for the fitting term (i.e., $n=5$ ) in eq. (11). Once the fitting parameters are obtained, the Gibbs free energy of mixing, $\Delta G_{\text {mix }}$, is described as

$$
\begin{aligned}
\Delta G_{\text {mix }}\left(X_{\mathrm{Fe}}\right)= & k_{B} T\left[X_{\mathrm{Fe}} \ln \left(X_{\mathrm{Fe}}\right)+\left(1-X_{\mathrm{Fe}}\right) \ln \left(1-X_{\mathrm{Fe}}\right)\right] \\
& +\sum_{i=o}^{n} \frac{A_{i} X_{\mathrm{Fe}}^{i+1}}{(i+1)} .
\end{aligned}
$$

Figure 3(b) shows the Gibbs free energy of mixing for solid and liquid phases of the $\mathrm{Fe}-\mathrm{Cr}$ alloy at $2500 \mathrm{~K}$. The (a)

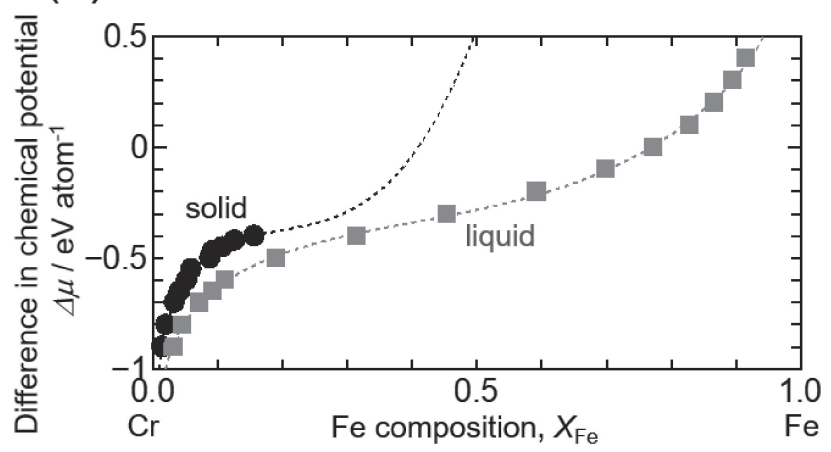

(b)

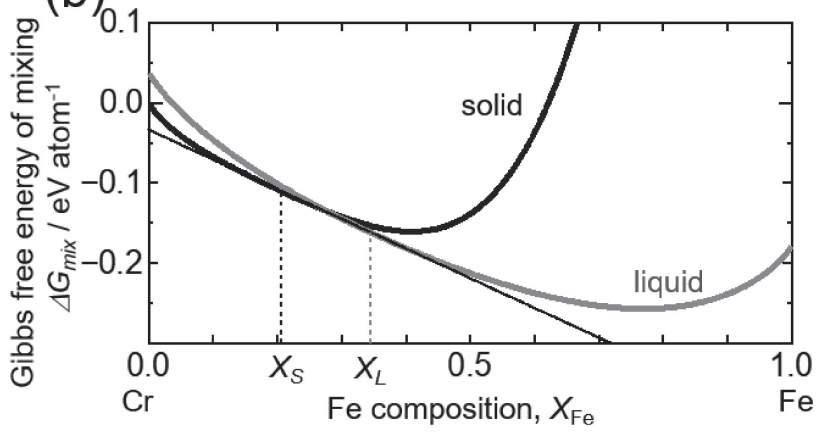

Fig. 3 (a) Relationship between the difference in chemical potential and the Fe composition for solid and liquid phases of $\mathrm{Fe}-\mathrm{Cr}$ alloy at $2500 \mathrm{~K}$ calculated by the semigrand canonical Monte Carlo (SGCMC) simulation. (b) Gibbs free energy of mixing for solid and liquid phases of the $\mathrm{Fe}-\mathrm{Cr}$ alloy at $2500 \mathrm{~K}$ obtained by thermal integration of the fitted lines in (a).

difference in the Gibbs free energy between the solid and liquid phases at $X_{\mathrm{Fe}}=0$ is separately estimated by integration of the Gibbs-Helmholtz relation ${ }^{50)}$ as

$$
\Delta G_{\mathrm{SL}}=T \int_{T}^{T_{M}} \frac{H_{L}\left(T^{\prime}\right)-H_{S}\left(T^{\prime}\right)}{T^{\prime 2}} d T^{\prime} .
$$

Since the enthalpy, $H$, is directly calculated from the MD simulation, $\Delta G_{\mathrm{SL}}$ can be derived straightforwardly. Finally, 

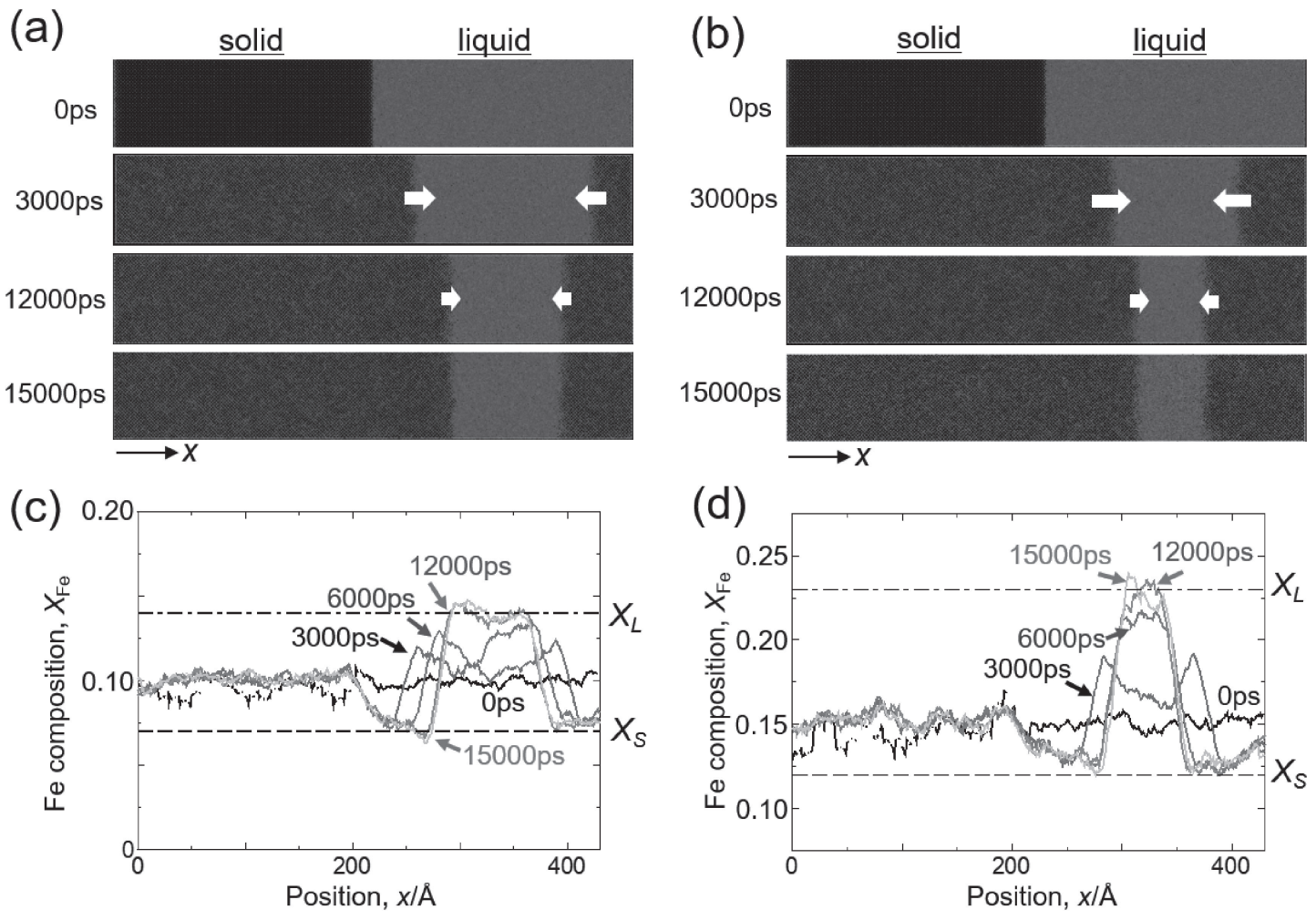

Fig. 4 (a)(b) Snapshots of relaxation of biphasic systems (a) $\mathrm{Cr}-10$ at $\% \mathrm{Fe}$ at $2500 \mathrm{~K}$ and (b) $\mathrm{Cr}-15$ at $\% \mathrm{Fe}$ at $2400 \mathrm{~K}$. (c)(d) Distribution of Fe composition in the biphasic systems (c) $\mathrm{Cr}-10$ at\%Fe $(2500 \mathrm{~K})$ and (d) $\mathrm{Cr}-15$ at\% Fe $(2400 \mathrm{~K}){ }^{54)}$

the solidus and liquidus compositions are derived from the contact points of the common tangent line. This technique is straightforward provided the particular value of $\Delta \mu$ is uniquely assigned to one composition as in an isomorphous alloy (i.e., all proportional solid solution). However, this technique is not applicable without modification when the relationship between $\Delta \mu$ and the composition is not monotonic, which causes a miscibility gap in the phase diagram. ${ }^{47)}$ A higher-order technique has been developed for cases including a miscibility gap. ${ }^{53)}$

Although the SGCMC simulation is a promising technique for estimating the solidus and liquidus compositions of a binary alloy, an MC simulation does not include the dynamics of the system in principle. Therefore, we employ a simple technique based on the MD simulation to estimate the solidus and liquidus compositions, in which the solute partition at the solid-liquid interface is monitored. ${ }^{54)}$ Figures 4(a) and 4(b) show snapshots of the relaxation process of the biphasic systems $\mathrm{Cr}-10$ at $\% \mathrm{Fe}$ at $2500 \mathrm{~K}$ and $\mathrm{Cr}-15$ at $\% \mathrm{Fe}$ at $2400 \mathrm{~K}$, respectively. The relaxation is performed for $15000 \mathrm{ps}$ under the NPT-constant condition. In both systems, solidification occurs in the initial stage and the solid-liquid interface stops moving after approximately 12000 ps. Figures 4(c) and 4(d) show the distribution of the Fe composition in each biphasic system during the relaxation. As the solidification proceeds, a solute $(\mathrm{Fe})$ partition develops at the solid-liquid interface, which reduces the Fe composition in the newly solidified region and increases the Fe composition in the liquid phase near the interface. After approximately $12000 \mathrm{ps}$, the solid-liquid interface stops moving and the distribution of the Fe composition becomes constant. Therefore, this distribution is considered as the

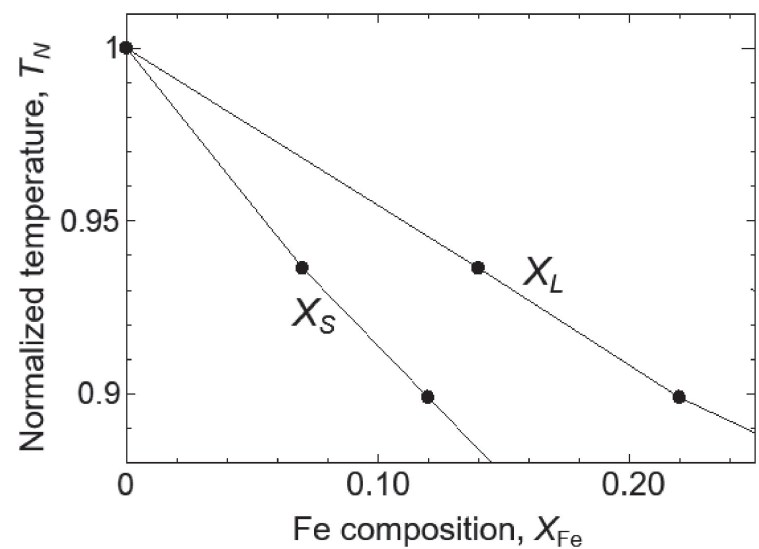

Fig. 5 Partial phase diagram of $\mathrm{Cr}$-rich area of $\mathrm{Fe}-\mathrm{Cr}$ alloy estimated by $\mathrm{MD}$ simulation of relaxation of $\mathrm{Fe}-\mathrm{Cr}$ biphasic system. The temperature is normalized by the melting point of $\mathrm{Cr}$ for the EAM potential ${ }^{52)}$ employed in the simulation.

equilibrium composition of the solid and liquid phases (i.e., solidus and liquidus compositions) at the calculated temperature. Figure 5 shows the partial phase diagram of the Cr-rich area of $\mathrm{Fe}-\mathrm{Cr}$ alloy estimated by MD simulation of the relaxation of an $\mathrm{Fe}-\mathrm{Cr}$ biphasic system. In the phase diagram, the temperature normalized by the melting point of $\mathrm{Cr}$ for this EAM potential is employed. By connecting these plots, solidus and liquidus lines are obtained.

\subsection{Grain boundary energy and grain boundary mo- bility}

Since most practical metals and alloys have a polycrystalline microstructure, it is essential to understand the properties 
(a)
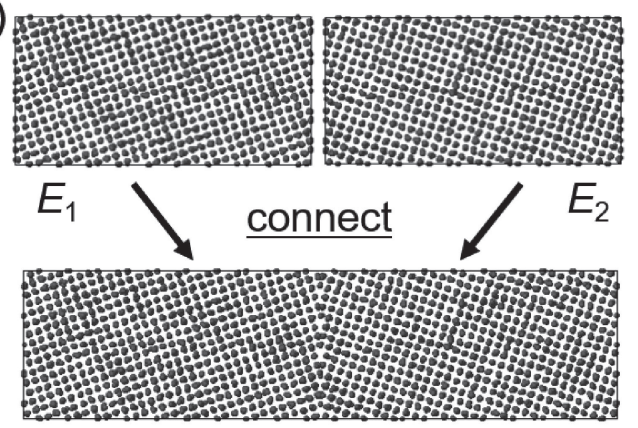

(b)
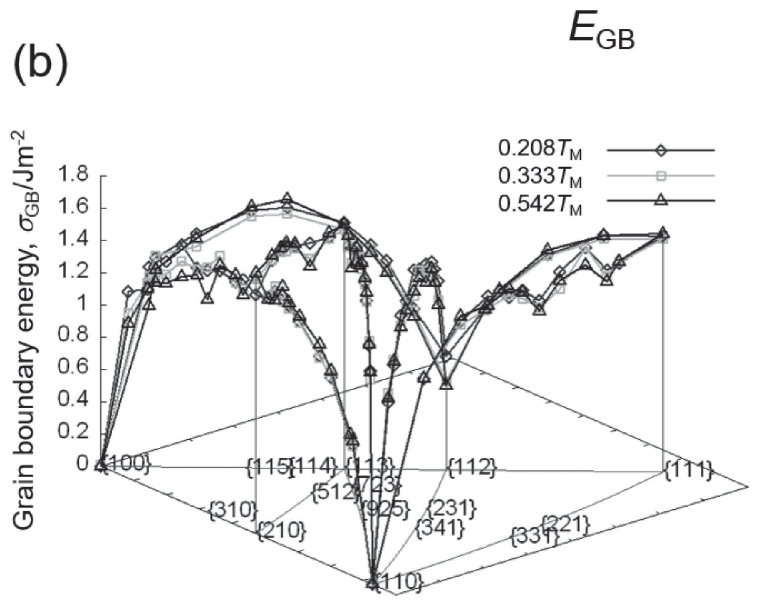

Fig. 6 (a) Schematic image of calculation of grain boundary energy in an MD simulation. (b) Grain boundary energy of the symmetric tilt boundary in bcc iron for various tilt axes. ${ }^{44)}$

of grain boundaries. Usually, the grain boundary energy $\sigma_{\mathrm{GB}}$ is estimated ${ }^{44,55-57)}$ from the difference between the energy of a bicrystal with a particular grain boundary $\left(E_{\mathrm{GB}}\right)$ and the total energy of the constituent crystals $\left(E_{1}+E_{2}\right)$ as

$$
\sigma_{G B}=\frac{E_{G B}-\left(E_{1}+E_{2}\right)}{2 A},
$$

where $A$ is the area of the grain boundary. Figure 6(a) shows a schematic image of the calculation procedure of the grain boundary energy. The energy difference is usually divided by $2 A$ owing to the three-dimensional periodic boundary condition. Figure $6(\mathrm{~b})$ shows the grain boundary energy of the symmetric tilt grain boundary in bcc $\mathrm{Fe}$ at a finite temperature calculated using the Finnis-Sinclair (FS) potential. ${ }^{58)}$ The figure is reconstructed from data in our previous study. ${ }^{44)}$ In the figure, the temperature is normalized by the melting point of bcc Fe by the FS potential $\left(T_{\mathrm{M}}=\right.$ $2400 \mathrm{~K})$. In general, the grain boundary energy increases with increasing temperature owing to the structural fluctuation caused by thermal vibration. ${ }^{44}$ A large energy cusp appears at the $(112)\langle 110\rangle \Sigma 3$ boundary, whereas no energy cusps appear at the (111) $\langle 111\rangle \Sigma 3$ boundary. Detailed information is given in our previous report. ${ }^{44)}$ The grain boundary energies of alloy systems can be estimated in the same manner. For example, the grain boundary energy of the symmetric tilt boundary in $\mathrm{Fe}-\mathrm{Cr}$ alloy ${ }^{57)}$ has been systematically calculated: the grain boundary energy basically decreases with increasing $\mathrm{Cr}$ composition. Recently, a virtual screening technique based on machine learning ${ }^{14}$ ) has been utilized to predict the optimum structure of a grain boundary among various possible structures, which is a promising technique for efficiently finding of the optimum structures of materials in the future.

In contrast to the grain boundary energy, it is not straightforward to explicitly estimate the grain boundary mobility by MD simulation since the planar grain boundary does not move unless a driving force acts on it. Therefore, a driving force is given in MD simulations such as curvature, ${ }^{59,60)}$ a triple junction, ${ }^{61,62)}$ shear stress, ${ }^{63-65)}$ or an additional interatomic potential. ${ }^{66,67)}$ The mobility of a specific grain boundary has been widely estimated using these techniques. However, it is not straightforward to evaluate the grain boundary mobility in a polycrystalline microstructure, in which many grain boundaries exist in a complicated manner. Moreover, it is considered that boundary migration is strongly affected by the roughness of the grain boundary. ${ }^{68)}$ Therefore, further studies are required to establish a technique for estimating the grain boundary mobility in a polycrystalline microstructure from the atomistic viewpoint.

\subsection{Solid-liquid interfacial energy}

The solid-liquid interfacial energy is one of the key factors determining the solidification microstructure, which is defined as the excess Gibbs free energy at the solid-liquid interface. ${ }^{69)}$ Although considerable effort has been made to measure the solid-liquid interfacial energy of metals and alloys over many years, ${ }^{69-71)}$ it is not yet straightforward to measure it with a high degree of accuracy. Therefore, the estimation of the solid-liquid interfacial energy by atomistic simulation is strongly desired. Several techniques have been proposed for estimating the solid-liquid interfacial energy by MD simulations. One of the popular techniques is the capillary fluctuation method. ${ }^{72,73)}$ In this technique, the interfacial stiffness is extracted from the capillary fluctuation spectrum of the roughness of the solid-liquid interface using the following relation: ${ }^{72,73)}$

$$
\left\langle\left|A(k)^{2}\right|\right\rangle=\frac{k_{B} T_{M}}{b W\left(\gamma+\gamma^{\prime \prime}\right) k^{2}},
$$

where $\gamma$ is the solid-liquid interfacial energy, $A(k)$ is the amplitude of the fluctuation of the solid-liquid interface with wavenumber $k$, and $W$ and $b$ are the length and thickness of the solid-liquid interface, respectively. The value $\tilde{\gamma}=$ $\gamma+\gamma^{\prime \prime}$ is called the interfacial stiffness. The anisotropic interfacial energy can be expressed by a low-order expansion consistent with cubic symmetry ${ }^{74}$ in the case of weak anisotropy $^{72)}$ as

$$
\begin{aligned}
\gamma= & \gamma_{0}\left[1+\varepsilon_{1}\left(\sum_{i} n_{i}^{4}-\frac{3}{5}\right)\right. \\
& \left.+\varepsilon_{2}\left(3 \sum_{i} n_{i}^{4}+n_{1}^{2} n_{2}^{2} n_{3}^{2}-\frac{17}{7}\right)\right],
\end{aligned}
$$

where $n_{\mathrm{i}}$ represent the orientation indices of the solid-liquid interface $\left(n_{1}, n_{2}, n_{3}\right)$, and $\varepsilon_{1}$ and $\varepsilon_{2}$ are the anisotropy parameters. Using eq. (16), the anisotropy in the solid-liquid interface can be extracted using the information on the solidliquid interface with the three different orientations. This is the merit of capillary fluctuation method. Moreover, a 
(a)
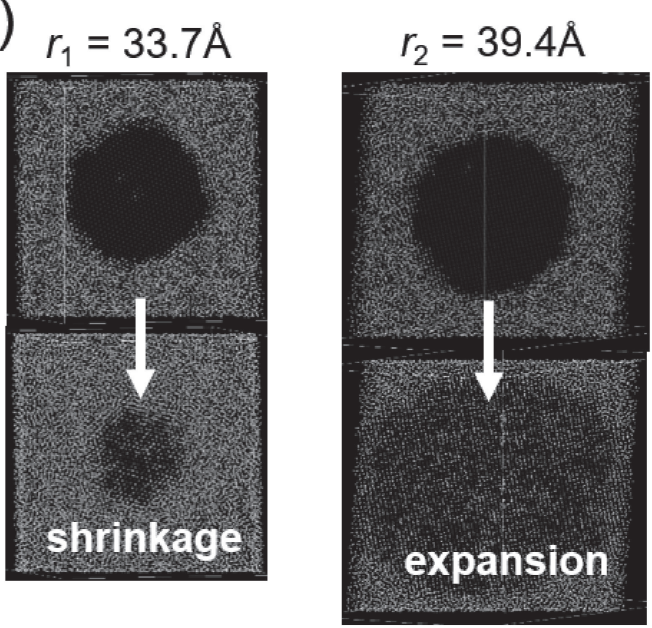

(b)

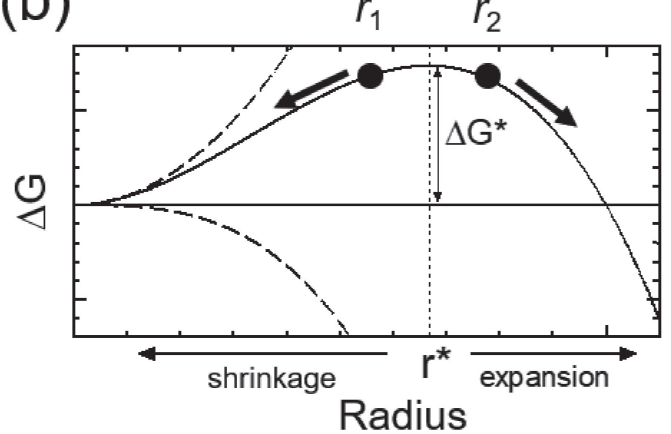

Fig. 7 (a) Snapshots of shrinkage or expansion of a spherical particle of bcc Fe placed in an undercooled iron melt $(\Delta T=110 \mathrm{~K}) .{ }^{79)}$ (b) Schematic image of the change in free energy as a function of particle radius according to classical nucleation theory.

cleaving technique has been developed for estimating the solid-liquid interface, in which thermodynamic integration is performed along a reversible path defined by the external cleaving potential. ${ }^{75,76)}$

Although the capillary fluctuation method is very useful for estimating the solid-liquid interfacial energy including the anisotropy, the result strongly depends on the definition of the solid-liquid interface in the atomistic configuration. ${ }^{77)}$ Therefore, we have estimated the solid-liquid interfacial energy using the Gibbs-Thomson effect in small solid particles in an undercooled melt, ${ }^{78-80)}$ in which the information on the solidliquid interface is not used explicitly. Figure 7(a) shows snapshots of the MD simulation ${ }^{79)}$ for the shrinkage or the expansion of a spherical particle of bcc Fe placed in an undercooled iron melt $(\Delta T=110 \mathrm{~K})$ using the FS potential. ${ }^{58)}$ The particle with a radius of $33.7 \AA$ shrinks in the undercooled iron melt of $\Delta T=110 \mathrm{~K}$, whereas that with a radius of $39.4 \AA$ expands in the iron melt of the same temperature. According to classical nucleation theory, ${ }^{81)}$ there is a critical radius of a spherical solid in an undercooled melt dividing the shrinkage and growth of the solid as illustrated in Fig. 7(b). Therefore, the critical radius is expected to be between 33.7 and $39.4 \AA$ for the iron melt of $\Delta T=110 \mathrm{~K}$. Practically, the critical radius for a target temperature is derived by changing the particle radius incrementally in repeated calculations. Then, the dependence of the temperature on the critical radius is also derived by changing the temperature systematically.

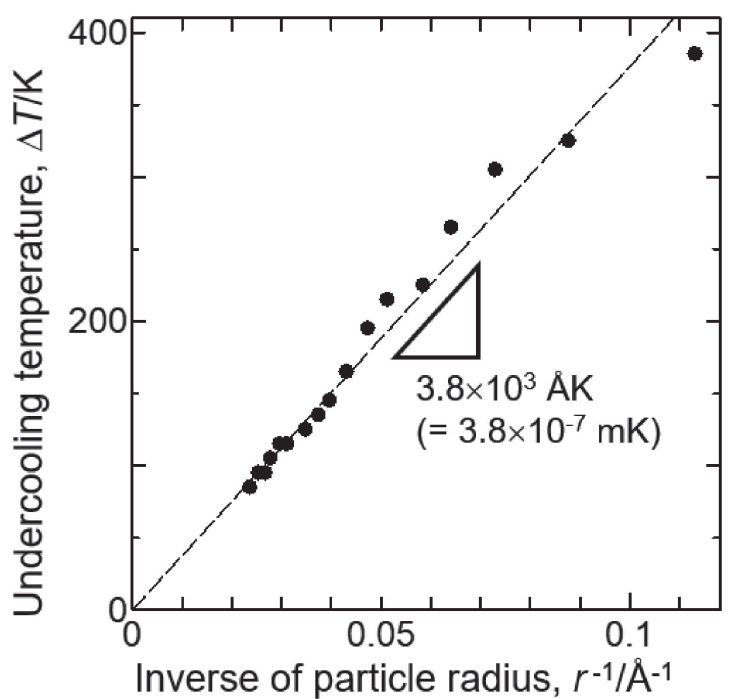

Fig. 8 Relationship between the undercooling temperature and the inverse of the critical radius obtained from by MD simulation of the melting of small solid particles in an undercooled melt for a pure Fe system. ${ }^{79)}$

Figure 8 shows the relationship between the undercooling temperature and the inverse of the critical radius obtained by MD simulations of the melting of small solid bcc particles in an undercooled melt for the Fe system using the FS potential. ${ }^{58)}$ The figure is reconstructed from data obtained in our previous study. ${ }^{79)}$ From classical nucleation theory, ${ }^{81)}$ the critical radius of a spherical solid in an undercooled melt, $r^{*}$, has the following inverse relation to the undercooling $\Delta T$,

$$
\Delta T=\frac{2 \gamma T_{M}}{\Delta H} \frac{1}{r^{*}},
$$

where $\Delta H$ is the latent heat. Therefore, the solid-liquid interfacial energy can be estimated from the slope of the relation in eq. (17). From the slope of the fitted line in Fig. 8, the solid-liquid interfacial energy of bcc $\mathrm{Fe}$ is estimated to be $1.7 \mathrm{~J} \mathrm{~m}^{-1}$ using $T_{M}=2400 \mathrm{~K}$ and $\Delta H=0.22 \mathrm{eV} /$ atom, which are values for pure $\mathrm{Fe}$ obtained using the FS potential. ${ }^{79)}$ A merit of this technique is that there is no need to define the solid-liquid interface explicitly from the atomistic configuration. On the other hand, the anisotropy in the solid-liquid interfacial energy cannot be defined using this technique and therefore only the average interfacial energy is estimated.

\subsection{Kinetic coefficient of solid-liquid interface}

The kinetic coefficient $\mu$ is defined as the proportional constant between the velocity of a solid-liquid interface, $v$, and the undercooling temperature $\Delta T$,

$$
v=\mu \Delta T \text {. }
$$

Since the anisotropy in the kinetic coefficient directly affects the morphology of the solidification microstructure, ${ }^{82,83)}$ it is essential to estimate the kinetic coefficient including its anisotropy. In an MD simulation, the kinetic coefficient is usually estimated from the temperature dependence of the propagation velocity of the planar solid-liquid interface in a biphasic system. ${ }^{79,84-86)}$ Figure 9(a) shows snapshots of the propagation of the solid-liquid interface with the (100) orientation in a solid-liquid biphasic system for bcc Fe at 


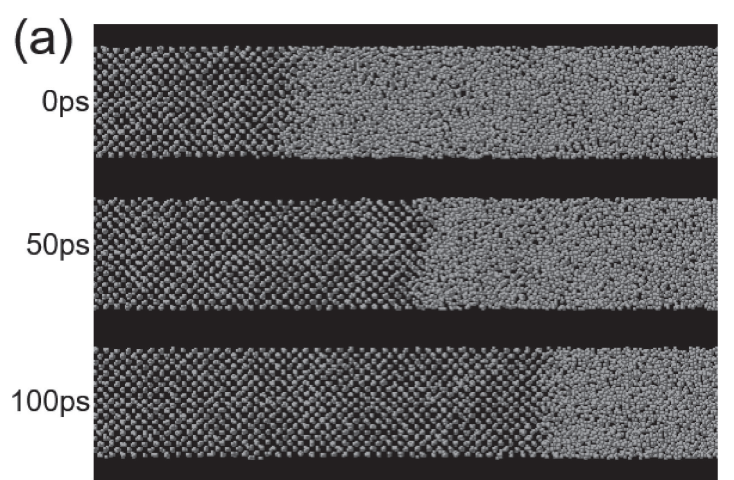

(b)

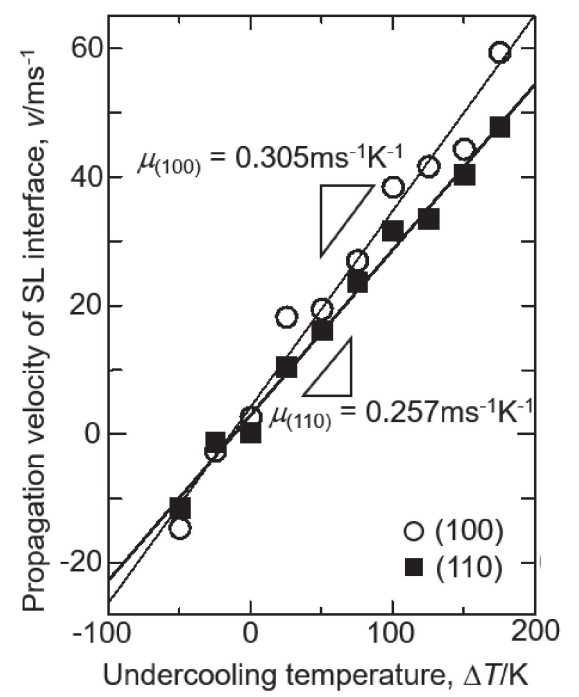

Fig. 9 (a) Snapshots of propagation of solid-liquid interface with (100) orientation in the solid-liquid biphasic Fe system at $\Delta T=100 \mathrm{~K}^{79}$ ) (b) Propagation velocity of the solid-liquid interface as a function of undercooling temperature.

$\Delta T=100 \mathrm{~K}$ using the FS potential. ${ }^{58)}$ The propagation velocity of the solid-liquid interface is directly estimated from the atomistic configuration. Figure 9(b) shows the propagation velocity of the solid-liquid interface as a function of undercooling temperature. The figure is reconstructed from data using our previous study. ${ }^{79)}$ From the slopes of the fitted lines, the kinetic coefficients for the (100) and (110) orientations are estimated as 0.305 and $0.257 \mathrm{~ms}^{-1} \mathrm{~K}^{-1}$, respectively. In general, the kinetic coefficient for the (100) orientation is higher than those for the (110) and (111) orientations, which causes variation in the morphology of the solidification microstructure. The kinetic coefficients for various metals and alloys estimated by MD simulations are summarized in the literature. ${ }^{87}$ )

\section{Summary}

In this paper, techniques for the estimation of thermodynamic and interfacial parameters from MD simulations are assessed by considering practical examples of the melting point of a pure metal, the solidus and liquidus compositions of a binary alloy, the grain boundary energy, the solid-liquid energy, and the kinetic coefficient. It is important to correctly understand how the coordinate and velocity of atoms obtained from an MD simulation are converted into thermodynamic and interfacial properties from a metallurgical viewpoint. Finally, I would like to mention our recent work, where the gap between MD and phase-field simulations is connected by direct mapping of the atomistic configuration of a polycrystalline microstructure obtained from an MD simulation into the phase-field property. ${ }^{88)}$ The cross-scale connection between atomistic and continuum scales is mainly a result of recent advance in highperformance computing. This direct mapping technique is expected to be applied to various numerical simulations of structural materials at the microstructure scale. ${ }^{89,90)}$ The provision of thermodynamic and interfacial parameters by MD simulations is strongly desired for the smooth connection between atomistic and continuum simulations.

\section{Acknowledgments}

This work was supported by a Grant-in-Aid for Scientific Research (B) (No. 16H04490) from Japan Society for the Promotion of Science (JSPS), Japan. Parts of this work were supported by the Council for Science, Technology and Innovation (CSTI), Cross-ministerial Strategic Innovation Promotion Program (SIP), "Structural Materials for Innovation" (funding agency: JST). The author thanks group member, Takuya Fujinaga and Kensho Ueno for the kind support in performing the simulations in Section 3.1 (T.F.), 3.2 (T.F.) and 3.3 (K.U.). The author also appreciates Munekazu Ohno and Hirono Minami of Hokkaido University for the fruitful discussion on the SGCMC simulation technique.

\section{REFERENCES}

1) Y. Shibuta, M. Ohno and T. Takaki: JOM 67 (2015) 1793-1804.

2) Y. Shibuta, M. Ohno and T. Takaki: Adv. Theor. Simul. 1 (2018) 1800065.

3) Y. Shibuta, S. Sakane, E. Miyoshi, S. Okita, T. Takaki and M. Ohno: Nat. Commun. 8 (2017) 10.

4) Y. Shibuta, K. Oguchi, T. Takaki and M. Ohno: Sci. Rep. 5 (2015) 13534.

5) Y. Shibuta, S. Sakane, T. Takaki and M. Ohno: Acta Mater. 105 (2016) 328-337.

6) S. Okita, E. Miyoshi, S. Sakane, T. Takaki, M. Ohno and Y. Shibuta: Acta Mater. 153 (2018) 108-116.

7) T. Zhu, J. Li, S. Ogata and S. Yip: MRS Bull. 34 (2009) 167-172.

8) T. Zhu, J. Li, A. Samanta, H.G. Kim and S. Suresh: Proc. Natl. Acad. Sci. USA 104 (2007) 3031-3036.

9) J. Song and W.A. Curtin: Acta Mater. 59 (2011) 1557-1569.

10) T. Shimokawa, T. Yamashita, T. Niiyama and N. Tsuji: Mater. Trans. 57 (2016) 1392-1398.

11) T. Tsuru, Y. Aoyagi, Y. Kaji and T. Shimokawa: Model. Simul. Mater. Sci. Eng. 24 (2016) 035010.

12) L. Zhang, C. Lu, K. Tieu and Y. Shibuta: Scr. Mater. 144 (2018) 78-83.

13) S. Okazaki and N. Yoshii: Computer Simulation no Kiso, 2nd ed., (Kagakudojin, Kyoto, 2011) pp. 235-255 (in Japanese).

14) S. Kiyohara, H. Oda, T. Miyata and T. Mizoguchi: Sci. Adv. 2 (2016) e1600746

15) Y. Hinuma, T. Hatakeyama, Y. Kumagai, L.A. Burton, H. Sato, Y. Muraba, S. Iimura, H. Hiramatsu, I. Tanaka, H. Hosono and F. Oba: Nat. Commun. 7 (2016) 11962.

16) M. Hanao, M. Kawamoto, T. Tanaka and M. Nakamoto: ISIJ Int. 46 (2006) 346-351.

17) M. Ohno, D. Kimura and K. Matsuura: Tetsu-to-Hagané 103 (2017) 711-719.

18) S. Ito, H. Nagao, A. Yamanaka, Y. Tsukada, T. Koyama, M. Kano and 
J. Inoue: Phys. Rev. E 94 (2016) 043307.

19) K. Sasaki, A. Yamanaka, S. Ito and H. Nagao: Comput. Mater. Sci. 141 (2018) 141-152.

20) M.P. Allen and D.J. Tildesley: Computer Simulation of Liquids, (Oxford University Press, Oxford, 1987) pp. 71-84.

21) R. Car, F. de Angelis, P. Giannozzi and N. Marzari: Handbook of Materials Modeling Part A, ed. by S. Yip, (Springer, Dordrecht, 2005) pp. 59-76.

22) Y. Shibuta, R. Arifin, K. Shimamura, T. Oguri, F. Shimojo and S. Yamaguchi: Chem. Phys. Lett. 565 (2013) 92-97.

23) T. Oguri, K. Shimamura, Y. Shibuta, F. Shimojo and S. Yamaguchi: J. Phys. Chem. C 117 (2013) 9983-9990.

24) S. Fukuhara, F. Shimojo and Y. Shibuta: Chem. Phys. Lett. 679 (2017) 164-171.

25) R. Sato, S. Ohkuma, Y. Shibuta, F. Shimojo and S. Yamaguchi: J. Phys. Chem. C 119 (2015) 28925-28933.

26) R. Sato, Y. Shibuta, F. Shimojo and S. Yamaguchi: Phys. Chem. Chem. Phys. 19 (2017) 20198-20205.

27) S. Sugiura, Y. Shibuta, K. Shimamura, M. Misawa, F. Shimojo and S. Yamaguchi: Solid State Ionics 285 (2016) 209-214.

28) J.E. Lennard-Jones: Proc. Phys. Soc. 43 (1931) 461-482.

29) P.M. Morse: Phys. Rev. 34 (1929) 57-64.

30) L.A. Girifalco and V.G. Weizer: Phys. Rev. 114 (1959) 687-690.

31) Y. Shibuta: Materia Japan 48 (2009) 61-66.

32) S. Daw and M.I. Baskes: Phys. Rev. B 29 (1984) 6443-6453.

33) W. Zhou, H.N.G. Wadley, R.A. Johnson, D.J. Larson, N. Tabat, A. Cerezo, A.K. Petford-Long, G.D.W. Smith, P.H. Clifton, R.L. Martens and T.F. Kelly: Acta Mater. 49 (2001) 4005-4015.

34) M.I. Baskes: Phys. Rev. B 46 (1992) 2727-2742.

35) F.H. Stillinger and T.A. Waber: Phys. Rev. B 31 (1985) 5262-5271.

36) J. Tersoff: Phys. Rev. B 37 (1988) 6991-7000.

37) D.W. Brenner, O.A. Shenderova, J.A. Harrison, S.J. Stuart, B. Ni and S.B. Sinnott: J. Phys. Condens. Matter 14 (2002) 783-802.

38) J.E. Mueller, A.C.T. van Duin and W.A. Goddard, III: J. Phys. Chem. C 114 (2010) 4939-4949.

39) T. Liang, T.-R. Shan, Y.-T. Cheng, B.D. Devine, M. Noordhoek, Y. Li, Z. Lu, S.R. Phillpot and S.B. Sinnott: Mater. Sci. Eng. R. 74 (2013) 255-279.

40) C.A. Becker, F. Tavazza, Z.T. Trautt and R.A. Buarque de Macedo: Curr. Opin. Solid State Mater. Sci. 17 (2013) 277-283 (http:// www.ctems.nist.gov/potentials)

41) S. Plimpton: J. Comput. Phys. 117 (1995) 1-19.

42) S.R. Phillpot, S. Yip and D. Wolf: Comput. Phys. 3 (1989) 20-31.

43) Y. Shibuta and T. Suzuki: Chem. Phys. Lett. 445 (2007) 265-270.

44) Y. Shibuta, S. Takamoto and T. Suzuki: ISIJ Int. 48 (2008) 1582-1591.

45) G.P. Purja Pun and Y. Mishin: Philos. Mag. 89 (2009) 3245-3267.

46) J. Wang, J. Li, S. Yip, D. Wolf and S. Phillpot: Physica A 240 (1997) 396-403.

47) D.R. Gaskell: Introduction to the Thermodynamics of Materials, 5th ed., (Taylor \& Francis Group, New York, 2008) pp. 263-303.

48) H. Ramalingam, M. Asta, A. van de Walle and J.J. Hoyt: Interface Sci. 10 (2002) 149-158.

49) J.J. Hoyt, J.W. Garvin, E.B. Webb, III and M. Asta: Model. Simul. Mater. Sci. Eng. 11 (2003) 287-299.

50) C.A. Becker, M. Asta, J.J. Hoyt and S.M. Foiles: J. Chem. Phys. 124 (2006) 164708

51) C.A. Becker, D.L. Olmsted, M. Asta, J.J. Hoyt and S.M. Foiles: Phys. Rev. B 79 (2009) 054109.

52) G. Bonny, R.C. Pasianot, D. Terentyev and L. Malerba: Philos. Mag. 91 (2011) 1724-1746.
53) B. Sadigh, P. Erhart, A. Stukowski, A. Caro, E. Martinez and L. Zepeda-Ruiz: Phys. Rev. B 85 (2012) 184203.

54) K. Ueno and Y. Shibuta: Materialia 4 (2018) 553-557.

55) D. Wolf: Philos. Mag. A 62 (1990) 447.

56) H. Nakashima and M. Takeuchi: Tetsu-to-Hagané 86 (2000) 357.

57) Y. Shibuta, S. Takamoto and T. Suzuki: Comput. Mater. Sci. 44 (2009) 1025-1029.

58) M.W. Finnis and J.E. Sinclair: Philos. Mag. A 50 (1984) 45-55.

59) M. Upmanyu, D.J. Srolovitz, L.S. Shvindlerman and G. Gottstein: Acta Mater. 47 (1999) 3901-3914.

60) H. Zhang, M. Upmanyu and D.J. Srolovitz: Acta Mater. 53 (2005) 7986.

61) M. Upmanyu, D.J. Srolovitz, L.S. Shvindlerman and G. Gottstein: Acta Mater. 50 (2002) 1405-1420.

62) Y. Shibuta, K. Oguchi and T. Suzuki: ISIJ Int. 52 (2012) 2205-2209.

63) H. Zhang and D.J. Srolovitz: Acta Mater. 54 (2006) 623-633.

64) J.W. Cahn, Y. Mishin and A. Suzuki: Acta Mater. 54 (2006) 49534975.

65) L. Zhang, C. Lu and Y. Shibuta: Model. Simul. Mater. Sci. Eng. 26 (2018) 035008.

66) K.G.F. Janssens, D. Olmsted, E.A. Holm, S.M. Foiles, S.J. Plimpton and P.M. Derlet: Nat. Mater. 5 (2006) 124-127.

67) D.L. Olmsted, E.A. Holm and S.M. Foiles: Acta Mater. 57 (2009) 3704-3713.

68) E.A. Holm and S.M. Foiles: Science 328 (2010) 1138-1141.

69) W. Kurz and D.J. Fisher: Fundamentals of Solidification, (Trans Tech Publication, Aedermannsdorf, 1998) pp. 21-44.

70) D. Turnbull: J. Appl. Phys. 21 (1950) 1022-1028.

71) M. Gündüz and J.D. Hunt: Acta Metall. 33 (1985) 1651-1672.

72) J.J. Hoyt, M. Asta and A. Karma: Phys. Rev. Lett. 86 (2001) 55305533.

73) J.R. Morris and X. Song: J. Chem. Phys. 119 (2003) 3920-3925.

74) W.R. Fehlner and S.H. Vosko: Can. J. Phys. 54 (1976) 2159-2169.

75) J.Q. Broughton and G.H. Gilmer: J. Chem. Phys. 84 (1986) 57595768.

76) R.L. Davidchack and B.B. Laird: Phys. Rev. Lett. 85 (2000) 47514754.

77) B. Cheng, G.A. Tribello and M. Ceriotti: Phys. Rev. B 92 (2015) 180102(R).

78) Y. Shibuta, Y. Watanabe and T. Suzuki: Chem. Phys. Lett. 475 (2009) 264-268.

79) Y. Watanabe, Y. Shibuta and T. Suzuki: ISIJ Int. 50 (2010) 1158-1164.

80) R. Hashimoto, Y. Shibuta and T. Suzuki: ISIJ Int. 51 (2011) 16641667.

81) J.A. Dantzig and M. Rappaz: Solidification, (EPFL Press, Lausanne, 2009) pp. 249-285.

82) Y. Shibuta, K. Oguchi and M. Ohno: Scr. Mater. 86 (2014) 20-23.

83) M. Guerdane and M. Berghoff: Phys. Rev. B 97 (2018) 144105.

84) J.J. Hoyt, B. Sadigh, M. Asta and S.M. Foiles: Acta Mater. 47 (1999) 3181-3187.

85) J.J. Hoyt, M. Asta and A. Karma: Interface Sci. 10 (2002) 181-189.

86) D.Y. Sun, M. Asta and J.J. Hoyt: Phys. Rev. B 69 (2004) 174103.

87) S.K. Deb Nath, Y. Shibuta, M. Ohno, T. Takaki and T. Mohri: ISIJ Int. 57 (2017) 1774-1779.

88) E. Miyoshi, T. Takaki, Y. Shibuta and M. Ohno: Comput. Mater. Sci. 152 (2018) 118-124.

89) E. Miyoshi, T. Takaki, M. Ohno, Y. Shibuta, S. Sakane, T. Shimokawabe and T. Aoki: npj Comput. Mater. 3 (2017) 25.

90) F. Briffod, T. Shiraiwa and M. Enoki: Mater. Sci. Eng. A 695 (2017) 165-177. 